\title{
Postabortion Family Planning: Addressing the Cycle Of Repeat Unintended Pregnancy and Abortion
}

\section{By Carolyn Curtis, \\ Douglas Huber and Tamarah Moss-Knight}

Carolyn Curtis is public health specialist/nurse midwife; team leader for postabortion care at the U.S. Agency for International Development, Washington, DC. Douglas Huber is senior reproductive health specialist at Innovative Development Expertise and

Advisory Services, Inc. (IDEAS), Newton,

MA. At the time this article was written, Tamarah MossKnight, a doctoral student at Howard University School of Social Work, was serving as a summer intern at USAID through the Global Health Fellows Program.
Some 35 million abortions occur in developing countries each year. Approximately 20 million of these are unsafe abortions, which claim the lives of 67,000 women as a result of related complications. These deaths represent 13\% of all pregnancy-related mortality and in some countries as much as $25 \%$ of maternal deaths. ${ }^{1}$ In developing countries, one of every 75 women die of pregnancy- or childbirth-related causes, compared to one of every 7,300 women in developed countries. ${ }^{2}$

If contraception were accessible and used consistently and correctly by women wanting to avoid pregnancy, maternal deaths would decline by an estimated $25-35 \%{ }^{3}$ Fifty-five million unintended pregnancies in developing countries occur every year to women not using a contraceptive method. Another 25 million occur as a consequence of incorrect or inconsistent use of a contraceptive method and method failure. ${ }^{4,5}$

\section{The Role of Postabortion Family Planning Services}

These statistics provide firm evidence that family planning reduces abortion, thus decreasing the risk of maternal death. In addition, over the last two decades, there has been increasing evidence that family planning interventions have a role to play not only before a woman has become pregnant, but after she has had an abortion or miscarriage.

In 1994, the international health community identified postabortion care (PAC) as an important strategy to reduce maternal mortality by treating complications related to unsafe abortion and miscarriage, and by providing postabortion family planning counseling and services to prevent repeat unplanned pregnancies and abortions. Although postabortion family planning counseling and service delivery is part of all postabortion care models, PAC services have historically sought to reduce maternal mortality by treating the symptoms of hemorrhage and sepsis rather than by treating women's unmet need for family planning, thus overlooking the potential of postabortion care to interrupt the cycle of repeat unplanned pregnancy, abortion and complications leading to maternal death.

For many postabortion patients, the lack of family planning counseling and services quickly leads to another induced abortion, because fertility returns within two to three weeks after miscarriage or induced abortion. This makes it essential to ensure that postabortion family planning counseling and service delivery are offered to all women who present for emergency obstetric or postabortion care, regardless of the method of treatment (sharp curettage, electric or manual evacuation) or place of treat- ment (operating theatre or PAC treatment room) as well as to all postpartum women.

\section{Postabortion Family Planning Service Models}

Postabortion family planning has been implemented in countries using two main methodologies. In countries where abortion is legal, programs offer postabortion family planning only. When women make an appointment for their abortion, they also receive family planning counseling. After their abortion has been completed, women are provided with family planning services before discharge from the facility.

In countries where abortion is illegal, emergency treatment and postabortion family planning counseling and services are provided as a single service. Both examples have resulted in increased family planning uptake and reduced repeat abortion. When deliberate efforts are made to strengthen the family planning component of postabortion care, improvements in family planning uptake can be realized within 12 months of implementation.

\section{Benefits of Postabortion Family Planning}

Regardless of the model used, providing postabortion family planning benefits individuals, families, communities and countries in many ways: increased modern contraceptive use and decreased abortion; reduced maternal and child mortality; prevention of mother-to-child HIV transmission and new HIV infections; and reduced social costs.

- Increased contraceptive use and decreased abortion. From 1991 to 1993, the Ministry of Health in Turkey, where abortion is legal, initiated a pilot program in 12 public and 12 private facilities to improve the provision of family planning to PAC patients. As a result of the pilot program, use of modern contraceptives among PAC clients increased from 65\% in 1991 to 97\% in 1992. From 1992 to 1998, this strategy was expanded to 10 additional large public hospitals. In 1999, the postabortion contraceptive acceptance rate was higher than $90 \%$ in the 10 public facilities. From 1998 to 2001, the overall rate increased from 37\% to $72 \%$ in 14 additional private hospitals. These interventions served as prototypes in the curriculum of Turkey's maternal and child health program. ${ }^{6}$

In the Women and Infant Health Project in Russia, where abortion is legal, family planning was strengthened for postpartum and postabortion women. Within four years (1999 to 2003), the number of women using a modern method of contraception increased by $16 \%$ (from $50 \%$ to $58 \%$ ). From 2003 to 2005, the number of abortions per 
1,000 women decreased from 49 to 43 per 1,000 women. ${ }^{7}$

In another study done in 2000 in Perm, Russia, researchers interviewed 1,516 women before and after they had an elective abortion procedure at one of five facilities using two interventions. The first intervention, at three hospitals, offered all postabortion clients predischarge family planning counseling and information, which were provided in a respectful, nonjudgmental manner. This was accomplished by training health care providers in family planning counseling and interpersonal communication skills and developing and supplying provider job aids and client-education materials on postabortion family planning. The second model, offered at two hospitals, used the same interventions, but also provided clients with a free initial supply of contraceptives. The proportion of women having a repeat abortion within 12 months was $10 \%$ at the study sites, compared with $20 \%$ in the general population. In addition, $67 \%$ of women given free contraceptives were still using a modern method after 12 months, compared with 53\% of those who did not receive free supplies. ${ }^{8}$

In Zimbabwe, where abortion is severely restricted, a 1996-1998 study comparing two hospitals showed that when the intervention site provided on-site counseling and access to free contraceptives at the same location and at the same time as emergency treatment, women were significantly more likely than women treated at the control site to adopt highly effective methods of contraception ( $96 \%$ vs. $5 \%$ ) and had fewer than half the unplanned pregnancies (42 vs. 96) during the year-long follow-up. The percentage of patients who had a repeat abortion during follow-up was more than twice as high at the control site, where only emergency services were provided, as at the intervention site (5.3\% compared to $2.5 \%$ ). ${ }^{9}$

Given the strong evidence that reorganizing services so that family planning counseling and service delivery are offered at the same time and in the same location as emergency treatment would improve family planning uptake, the Red Cross Hospital in Phnom Penh, Cambodia, conducted a pilot program incorporating these changes. Family planning counseling rose slightly from $96 \%$ to $100 \%$, while contraceptive uptake prior to hospital discharge increased from $51 \%$ to $90 \%$. The key change was that family planning services were offered to women in the same room, rather than referring them to another location in the hospital. On the basis of these findings, a reorganization of services was approved for regional hospitals in Pursat and Siem Riep. ${ }^{10}$ At a private nongovernmental organization in Cambodia, the contraceptive acceptance rate increased from $7 \%$ to $30 \%$ in one year after the family planning component of PAC services was strengthened. ${ }^{11}$

- Reduced child mortality. Ensuring that every woman seeking PAC services because of complications of unsafe abortion or miscarriage is offered family planning counseling and a contraceptive method before discharge from the facility will help to decrease repeat unplanned pregnancy and unsafe abortion. ${ }^{12}$ Moreover, postabortion family planning assists in reducing child mortality. Children whose mother dies are much more likely to be ill and die themselves than children whose mother remains alive. ${ }^{13}$

- Prevention of mother-to-child transmission of HIV and new infections. Postabortion family planning is also important for women who are HIV-positive. These women have a greater risk of miscarriage than HIV-negative women, and also risk passing their infection to their child during gestation or through breast-feeding. Improving voluntary postabortion contraception for this group of women will allow them to consider their own health and desire for further childbearing in light of their HIV status. Through the promotion of dual protection via postabortion family planning counseling and service delivery, new HIV infections as well as unintended pregnancies can be prevented.

- Reduced social costs. The costs of unsafe abortion extend well beyond those of the health system. Societies bear the economic cost of lower productivity caused by long-term disability, and mortality due to unsafe abortion. It is estimated that these additional costs are three or more times as great as health systems' cost for PAC, including the family planning component. ${ }^{14}$ In a study conducted of one PAC service model in Oaxaca, Mexico, the estimated cost per patient of providing family planning counseling and a contraceptive method totaled US $\$ 2.90,{ }^{15}$ compared with \$35-180 per patient for another PAC visit due to repeat unplanned pregnancy and abortion. ${ }^{16}$

\section{Barriers to Postabortion Family Planning}

A 2001 global evaluation of postabortion care programs found that in the delivery of postabortion care services, the second component of PAC (family planning counseling and services) did not receive as much attention as the first component (emergency treatment). ${ }^{17}$

A World Bank report recommended that postabortion care be strengthened by ensuring contraceptive counseling and that family planning services be provided in the same facility where uterine evacuation was performed to decrease opportunity costs. ${ }^{3}$ Situational analyses conducted in the Latin American and Caribbean region support these statements. ${ }^{18-20}$ The findings of the 2001 USAID global evaluation report, situational analyses in Latin America and the Caribbean and country action plans from Bolivia, Kenya and Senegal identified barriers for postabortion family planning that need deliberate and continued attention at various levels. ${ }^{17-20}$

At the national level, barriers to providing postabortion family planning include lack of sufficient financing for family planning in the national budgets, lack of adequate training, lack of supportive supervision and lack of availability of PAC services at health centers and other lower-level facilities.

At the facility level, barriers to postabortion family planning services fall into two categories.

- Guidelines, staffing, patient education materials (IEC). Lack of written guidelines for family planning in hospitals; insufficient staff for family planning counseling and service delivery; lack of IEC materials for client education; and lack of access to contraceptive methods during the af- 
ternoon, night and weekend shifts.

- Contraceptive methods. Lack of availability of contraceptive methods at the service delivery point, even when contraceptives are available in the facilities; lack of availability of a wide range of methods, thus limiting client choice; and lack of record-keeping systems that serve as reminders to use the oldest stocks first, thereby preventing the expiration of methods before they are used, and of systems that note whether women receiving PAC services receive family planning counseling and methods before they are discharged from the facility.

At the provider level, barriers include a lack of knowledge about the early return to fertility; denial of certain or all methods to some groups, such as adolescents or women who have not yet delivered a child; a lack of patient counseling on PAC procedure, on timing of return to fertility, and on choosing and obtaining a family planning method before discharge from the facility.

At the client level, obstacles to use of postabortion family planning services include fear of side effects; a belief that the risk of becoming pregnant is low, due to age, infrequent sexual intercourse or other reasons; lack of awareness or knowledge of family planning methods; and partner disapproval of contraceptive use.

\section{Promising Trends}

- Decentralization. The decentralization of services from tertiary hospitals to health center and health post levels has been successful in increasing the uptake of postabortion family planning. From 2003 to 2005, postabortion care services, which had previously been provided only in tertiary hospitals, were instituted in rural and urban health centers and health posts in Senegal. The proportion of women who received contraceptive counseling before leaving the facility increased from 36\% to $78 \%$ between the two years. Although baseline data on postabortion contraception acceptance was lacking, 56\% received a method before going home in 2005-an impressive rate in the context of West Africa. ${ }^{21}$

Decentralization of PAC services in Tanzania resulted in more than $95 \%$ of PAC clients treated at health centers receiving family planning counseling there as well, and an increase from $89 \%$ to $97 \%$ in the proportion of clients who accepted a method before leaving the facility. In the district hospital, health centers and dispensaries that did not have the strengthened family planning services at the site of PAC treatment, only $41 \%$ of clients adopted contraceptives. At dispensaries that had not strengthened their family planning services, only $14 \%$ of clients left with a method. ${ }^{21}$

- Community mobilization. Since 2004, PAC community mobilization activities implemented in Bolivia, Kenya and Senegal have increased knowledge of family planning methods, raised awareness of complications related to unsafe abortion and reduced stigma regarding postabortion care. ${ }^{22}$ These consisted of three eight-hour sessions on the causes of and means of preventing unsafe abortion, health care services available and dialogue to improve care and help reduce un- safe abortion and miscarriage. In Bolivia, families became aware of national insurance coverage for PAC services through community mobilization. The communities have advocated for provider training, improved quality of services, better relationships with health care providers, improved roads and bridges to increase access to care and new or better facilities for PAC and other health services.

In Bolivia, baseline and endline family planning statistics at health facilities were not collected as part of the program; however, preintervention and postintervention studies on knowledge, attitudes and practices (KAP) were conducted. Women did not seek family planning services because they lacked detailed knowledge about methods, were shy and worried about privacy and confidentiality, and were concerned that they would not be treated with respect. They were also unaware that the government provided health insurance. Adolescents, in particular, did not request reproductive health services as a result of these worries. ${ }^{22}$

Preintervention and postintervention KAP studies indicated an increased knowledge of contraceptive methods. Knowledge of the existence of contraceptive methods increased from $83 \%$ at baseline to $92 \%$ in the postintervention study. Women stated that they learned about the benefits of child spacing, about available methods, and how to obtain and use them. They also reported that they felt comfortable requesting family planning services because they were treated respectfully. ${ }^{22}$

In 2005, the PAC community mobilization program implemented in Bolivia was replicated in Kenya. In the year after community mobilization efforts were initiated, family planning visits by both PAC clients and the general population increased. New contraceptive visits at participating health facilities increased by more than $60 \%(8,500$ to 13,800 ), and follow-up visits more than doubled (from 2,000 to 4,300 ). These results may be due in part to the formation of community action plans that increased access to PAC and family planning services. This included emergency transport plans, PAC payment strategies, and leveraging of funds from local governments to build roads, bridges and new dispensaries. Funding from private donors and community contributions paid for provider training and necessary equipment. As a result of these interventions, USAID/Kenya is promoting this model for other provinces. $^{23}$

Pre- and posttest KAP scores for community participants in Senegal's PAC Community Mobilization program resulted in an increase in knowledge of the advantages of family planning (35\% to 65\%); an increase in contraceptive practice ( $27 \%$ to $62 \%$ ); a higher proportion of young mothers making decisions on their desired number of children (56\% to $60 \%$ ) and a reduction in the perception that family planning is a concern only for women (61\% to $51 \%){ }^{23}$

\section{Best Practices}

In 2004, more than 400 articles on postabortion care dating from 1994 to 2004 were assessed using Gray's five levels of evidence to determine the most effective treatment 
approaches. Strong evidence suggested that the following interventions increased postabortion family planning counseling and uptake, and therefore should be integrated into PAC programs: ${ }^{12}$

- Offer family planning counseling and methods at the same location where the woman receives emergency treatment.

- Counsel women who have had a miscarriage and want to get pregnant soon to wait at least six months before trying to conceive. This delay reduces the incidence of maternal anemia, premature rupture of membranes, low birth weight and preterm delivery in the next pregnancy.

- Inform male partners, when the woman agrees, about her condition, treatment, follow-up care and family planning method. Include male partners in family planning counseling as the woman desires.

- Train midwives to counsel PAC patients on family planning, STIs/HIV and nutrition, as well as to perform other aspects of PAC, including emergency treatment.

- Use dedicated family planning nurse counselors rather than referrals to other organizations for contraceptive services; this helps increase immediate contraceptive acceptance for PAC patients.

- Conduct contraceptive technology updates and counseling workshops for providers at the primary level to reinforce skills in postabortion family planning counseling and method provision prior to discharge.

\section{Program Implications}

Program goals for the future should include universal access to and, when desired, provision of family planning services following abortion, whether induced (safe or unsafe) or spontaneous. The need for at least a six-month pregnancy interval after miscarriage before attempting to become pregnant again guides providers to recommend family planning for all women receiving PAC services. Postabortion and postpartum family planning for all women go hand in hand, though there are some differences in appropriate contraceptive methods.

- Facilities and providers. To help women who want no more children avoid unwanted pregnancy, family planning providers should make sure that they offer highly effective long-acting and permanent methods, as well as reversible methods suitable for birth spacing. For example, programs need to retrain clinicians to provide counseling on and insertion of IUDs, particularly the Copper T 380, which is effective for 12 or more years. Given the favorable safety profile of copper IUDs, they deserve greater emphasis in postabortion and postpartum services.

Reorganization of services using simple delivery systems, job aids, efficient and effective counseling, and written instructions will be required for health systems to provide contraceptives for all clients regardless of the surgical, medical and anesthesia regimens used in PAC treatment. Written posttreatment instructions and contraceptive information are necessary in addition to verbal instruction, given the physical and emotional stress surrounding abortion and the inability to remember new information com- mon with antianxiety and narcotic pain medications. Women who are not literate generally have access to family members or neighbors who can read them the written instructions.

Postabortion uptake of family planning is needed just as much and can be as successful for women treated with sharp curettage under general anesthesia as for those treated with vacuum aspiration under local anesthesia. ${ }^{24,25}$ Therefore, reorganization of services is also needed in facilities where women are treated in the operating room.

- Policymakers. Policies and funding decisions need to emphasize postabortion family planning needs. Providing sufficient resources for postabortion family planning from national budgets and international donors is essential. These resources include not only the training for postabortion care services, but family planning commodities and other equipment needed to provide the package of postabortion care services, which must be included in national, regional, district and facility budgets. Training for in-service and preservice providers should reflect the new priorities. Accordingly, job descriptions will need to change so that the scope of work is inclusive of postabortion family planning counseling and service delivery.

- Professional organizations. Organizations such as the International Confederation of Midwives (ICM), the International Federation of Gynecologists and Obstetricians (FIGO) and the International Council of Nurses (ICN) should jointly engage policymakers, their members and the institutions where they work to effect change. In many cases, these professionals are in the best position to influence policy and the priorities for health resources in their countries and the scope of practice for their members. Decentralization of services to midlevel clinicians will require collaboration and teamwork among medical, nursing and midwifery providers and professional organizations. As a means of bringing attention to the urgency of the need to strengthen postabortion family planning, a joint statement on postabortion family planning was endorsed by FIGO, ICM, ICN and USAID in September 2009. ${ }^{26}$

- Faith-based organizations. According to a May 2008 survey of Christian health organizations, many faith-based organizations want to strengthen family planning services and respond to the gap in contraceptive services, especially in Africa, including family planning for postabortion care clients. Since faith-based organizations provide $30-70 \%$ of health care in many African countries, they have the potential to increase the uptake of family planning among postabortion clients and reduce unmet need for family planning and repeat unsafe abortion. ${ }^{27}$

- Monitoring and evaluation. Contraceptive prevalence rates are critical health measures of family planning program performance. A key output measure of postabortion family planning performance is the proportion of women leaving the facility with a method. This indicator has increased rapidly in postabortion facilities and programs when family planning services are strengthened. Important measures of quality for PAC programs will document 
that women received family planning counseling, that women received concise written instructions for using their chosen method, that women have a plan for followup family planning services, that there is involvement of husbands and partners when the woman agrees, and that a plan for ongoing contraceptive supplies exists.

\section{CONCLUSIONS}

Postabortion family planning has always been a key element of PAC and provides a powerful rationale for PAC services. In practice, however, treatment of complications is usually the sole focus and family planning is often neglected. It is essential to restore the family planning component to PAC services, not only to prevent repeat unintended pregnancy and abortion, but also because it is integral to achieving the Millennium Development Goals, UNGASS goals for HIV and other health objectives-reducing maternal morbidity and mortality, mother-to-child transmission of HIV and new HIV infections. Some of the barriers identified here can be overcome by using the free, evidence-based, online tools at <www.postabortioncare. org $>$ and those of other agencies. Given the examples we have presented of how services can produce cost savings as well as rapid results, women and communities should demand quality PAC services that provide them with accessible, cost-effective postabortion family planning services that can assist them in reducing unplanned pregnancy and repeat abortion, reduce new HIV infections, improve the health of a woman's next child and ultimately improve the health of her family.

\section{REFERENCES}

1. World Health Organization (WHO), Unsafe Abortion; Global and Regional Estimates of the Incidence of Unsafe Abortion and Associated Mortality in 2003, fifth ed., Geneva: WHO, 2007.

2. WHO, Maternal Mortality in 2005; Estimates Developed by WHO, UNICEF, UNFPA and the World Bank, Geneva: WHO, 2007.

3. Lule E, Singh S and Chowdhury SA, Fertility Regulation Behavior and Their Costs: Contraception and Unintended Pregnancies in Africa and Eastern Europe and Central Asia, Washington, DC: World Bank, 2007.

4. Sedgh $G$ et al., Women with an unmet need for contraception in developing countries and their reasons for not using a method, Occasional Report, New York: Guttmacher Institute, 2007, No. 37.

5. Singh S et al., Adding It Up: The Benefits of Investing in Sexual and Reproductive Health Care, New York: Guttmacher Institute and UNFPA, 2003.

6. Senlet $\mathrm{P}$ et al., Bridging the gap: integrating family planning with abortion services in Turkey, International Family Planning Perspectives, 2001, 27(2):90-95.

7. Cappa L et al., Russia: Integrating Family Planning into the Health System: A Case Study of the Maternal and Child Health Initiative, Arlington, VA: John Snow, 2007.

8. Savelieva I et al., Postabortion Family Planning Operations Research Study in Perm, Russia, Washington, DC: Population Council, 2003.

9. Johnson BR et al., Reducing unplanned pregnancy and abortion in Zimbabwe through postabortion contraception, Studies in Family Planning, 2002, 33(2):195-202

10. Nasy S, Postabortion family planning: using results to advocate for integrated services, Powerpoint presentation at Asia and Near East (ANE) Best Practices Meeting, Bangkok, Thailand, Sept. 6, 2007.

11. Ping. C. Best practices for postabortion family planning: RHAC's experience, Powerpoint presentation at the ANE Best Practices Meeting, Bangkok, Thailand, Sept. 6, 2007.

12. U.S. Agency for International Development (USAID), What Works: A Policy and Program Guide to the Evidence on Family Planning, Safe Motherhood, and STI/HIV/AIDS Interventions-Module 2: Postabortion Care, Washington, DC: USAID, 2007.

13. UNFPA, Focus on 5. Women's health and the MDGs, $<$ https://www.unfpa.org/webdav/site/global/shared/images/publication s/2009/focus_on_5.pdf>, accessed Mar. 16, 2010,

14. Vlassoff $\mathrm{M}$ et al., Economic impact of unsafe abortion-related morbidity and mortality: evidence and estimation challenges, Research Report No. 59, <http://www.ids.ac.uk/ids/bookshop/rr/Rr59.pdf>, accessed Mar. 15, 2009.

15. Brambila C et al., Estimating costs of postabortion services at Dr. Aurelio Valdivieso General Hospital, Oaxaca, Mexico, in: Huntington D and Piet-Pelon N, eds., Postabortion Care: Lessons from Operations Research, New York: Population Council, 1999.

16. Hamilton M, Is there value in adding a second visit to postabortion care? Washington, DC: The Policy Project, 2006.

17. Cobb L et al., Global evaluation of USAID's postabortion care program, Washington, DC: USAID, 2001.

18. Fuentes ER et al., Situation analysis of the integration of family planning services in postpartum, postabortion, and prevention of mother to child transmission programs in Haiti, Washington, DC: Population Council, 2008

19. Quitero G et al., Situation analysis of the integration of family planning services in postpartum, postabortion, and prevention of motherto-child transmission programs in the Dominican Republic, Washington, DC: Population Council, 2008.

20. Solis F et al., Situation Analysis of the Integration of Family Planning Services in Postpartum, Postabortion, and Prevention of Mother-to-Child Transmission Programs in Nicaragua, Washington, DC: Population Council, 2008.

21. Curtis C, Decentralization of postabortion care services in Senegal and Tanzania, Global Health Technical Briefs, Washington, DC: USAID, 2007

22. Curtis C, What the community is telling us about their needs in PAC: Synthesis of Phase I community action plans from Bolivia, Senegal, and Kenya, presentation at Moving Forward with Postabortion Care: Lessons Learned from Five Postabortion Care Focus Countries, Mar. 18, 2008, Washington, DC.

23. The ACQUIRE Project, Community Postabortion Care Projec (COMMPAC) in Nakuru District, Kenya: Summary Report, Phase I July 2005-September 2006, New York: The ACQUIRE Project/ EngenderHealth.

24. Billings DL, Fuentes VJ and Perez-Cuevas R, Comparing the quality of three models of postabortion care in public hospitals in Mexico City, International Family Planning Perspectives, 2003, 29(3):112-120.

25. Rasch V, Huber D and Akande EO, Report of the Postabortion Care Technical Advisory Panel, USAID, Washington, DC: USAID, 2007.

26. International Federation of Gynecology and Obstetrics (FIGO), International Confederation of Midwives (ICM), International Counci of Nurses (ICN) and USAID, Family planning: a key component of post abortion care, consensus statement, Washington, DC: FIGO, ICN, ICN and USAID, 2009.

27. Huber D et al., International Family Planning: Christian Actions and Attitudes, McLean, VA: Christian Connections for International Health, 2008

\section{Acknowledgments}

The authors wish to thank the U.S. Agency for International Development for supporting Carolyn Curtis's work on this article, the Extending Service Delivery Project for providing time for Douglas Huber to coauthor the article and Jeffrey Spieler for his review of an early draft.

Author contact: ccurtis@usaid.gov 